\title{
Technical Conception of Ghosting Images for Windows Platforms
}

\author{
Shadreck Mudziwepasi \\ Computer Science Department \\ University of Fort Hare \\ P-Bag X1314, Alice 5700, RSA
}

\author{
Mfundo Shakes Scott \\ Computer Science Department \\ University of Fort Hare \\ P-Bag X1314, Alice 5700, RSA
}

\author{
Nogwina Mnoneleli \\ Computer Science Department \\ University of Fort Hare \\ P-Bag X1314, Alice 5700, RSA
}

\begin{abstract}
Global advances in Information Technology (IT) have led to the adoption of computer systems in organizations and institutions alike all over the world. This trend has led to the introduction of many computer applications and softwares that are mearnt to perform specific tasks so long they are supported by the Operating Systems (OSs) installed in Personal Computers (PCs). It is however important to note that these applications and associated softwares are released in different versions and may also come with different addons as per different versions and upgrades. This requires that we continuously upgrade our computers and associated systems so that they become compatible with new versions of different softwares that are released almost each and every single day. Some computers might actually crash, thereby requiring re-installment of all applications there-on before we assume a system restore point. Continuous upgrade and maintenance of such computers and their systems is imminent. However, for a large institution that may possess thousands of PCs in their system, it might be difficult to perform PC by PC upgrade and maintenance. We can therefore create ghosting images for windows and open source platforms. Ghosting a computer basically involves making use of a Ghosting Server to copy all the contents of a computer's hard drive including the OS. These would be stored as ghosting images. Thus, for computers that will be sitting on the same sub network with the Ghosting Server as their domain controller, we can then perform an upgrade and maintenance routine on all of them at once. Therefore, the process of ghosting images deployment would provide convenience in performing installations and upgrades for organizations and large institutions in a cost and time effective manner.
\end{abstract}

\section{General Terms}

Ghosting Server, Operating System, Domain Controller and Personal Computers

\section{Keywords}

Ghosting images, Windows and Open Source Platforms.

\section{INTRODUCTION}

This paper presents a thorough outline of how ghosting images are created, further to which it also provides an exhaustive explanation of how the available ghosting softwares operate. Examples of such software include the Windows Deployment Services (WDS), Norton ghost software otherwise referred to as Symantec ghost software and the Personal Computer (PC) Disk Clone X [1]. Through implementation of these and other such softwares available, we can be able to copy all the contents of a hard drive including the OS from any PC onto any secondary storage device. The storage media may be a Compact Disk(CD), Universal Serial Bus (USB) or any specific server in a case were we would need to deploy the stored softwares and applications as ghost images into PCs that may be connected onto a particular sub-network. This form of computing may therefore prove to be vital for large institutions and organizations that house plenty of PCs which overally may duely be performing the same task and offering the same services to specific users. Examples of such include Vodacom South Africa were there are plenty of PCs housed in different computer laboratories and offices, though however, all of them would be performing the same functions and may actually be needing the same applications to achieve the overall business goal of the entity. We can also note some educational institutions such as Oxford University in the United Kingdom (UK) where a large number of PCs may need to be installed with the same applications for them to offer assigned services to end-users which can be the staff members or students of this University [2]. We can also solve the complication that can arise from differing needs and operations between staff members and students by creating two images. Each image would be for the two different domains with different specifications to cater for the different services required and the operations performed by either of the user in each domain. Henceforth, we would only need to boot the PCs from the network all at once and deploy the required image subsequently installing a specific application in all networked PCs at once. Furthermore, ghosting can also help in cases of storage media failures or virus attacks. These scenarios may require formatting of the hard drive or any affected storage media which may be a USB as an example. We would therefore need not to worry about the loss of data as the ghost image we would have created can provide us with a solution for such a backup purpose. In this paper, we hereby explore the different techniques used to create ghosting images and thoroughly investigate the background functioning processes of a ghosting software with the aim of giving a clear illustration of how ghosting softwares function.

\section{CREATING A SOURCE COMPUTER}

This is the stage where all the softwares and applications including the operating system needed are installed in one computer and cloned or copied. The term that is given for this stage is called "creating the Source Computer"; meaning it is the computer that contains all the software and applications required for the new computers or for the computer that needs fixing [4].

\section{GHOSTING A COMPUTER}

Ghosting implies creating an identical image of hard drives where all the information in the source computer is copied and installed in the new computer [5]. The steps followed to start this process when using symantec ghosting software are outlined in the figure below: 


\begin{tabular}{|c|c|}
\hline START & $\begin{array}{l}\text { - The user clicks starts from the ghosting } \\
\text { server where symantec ghost is installed }\end{array}$ \\
\hline ALL PROGRAMS & $\begin{array}{l}\text { - Then from the installed programs select } \\
\text { symantec ghost. }\end{array}$ \\
\hline SYMANTEC GHOST & $\begin{array}{l}\text { - symantec ghost selected and ready to } \\
\text { procced with the creation of images. }\end{array}$ \\
\hline
\end{tabular}

Figure 1: Opening Symantec Ghost

\section{GHOSTING SOFTWARE}

There are many ghosting softwares available on the Information Technology (IT) market today. Each has its own advantages and shortcomings. We used symantec ghosting software for this project. This is because Symantec Ghost Solution Suite is the industry's most widely used PC management solution with ease-of-use for managing the entire PC lifecycle including OSs deployment, software distribution, PC migration and retirement [6]. The Solution Suite reduces IT costs by streamlining networked desktop and laptop management in an enterprise environment. It provides both file-based and sector-based imaging technology, hardware and software inventory, a client staging area, and multicast file transfer to enable fast and easy PC management and secure data disposal.

\section{NORTON GHOST SOFTWARE}

Ghosting is a hybrid idea which has two layers, the partition layer on which it deals with partition resizing and the file system layer on which it deals with filing present on the file system. Symantec Ghost added support for the NTFS file system in 2006, and also provided a program, Ghostwalker (DOS name: ghstwalk.exe), to change the Security ID (SID) that made Windows NT Version 4.0 which also moved from real-mode DOS 286 to protected-mode and the additional memory available allowed for the introduction of Ghost 6.0 which included a console [7]. This would simplify the management of large numbers of machines. The Console communicates with client software on managed computers to allow a system administrator to refresh the disk of a machine remotely. As it needs to understand the file system structure Norton Symantec Ghost performs much more complex operations. Since sector-by-sector image-based backup like classic Unix Data Description (DD) only copies raw data, usually backups are made using this method which has "all-ornothing" property, without special drivers (loopback drivers) they cannot be used for the restoration of a single file or directory.

\section{CREATION OF THE BOOT DISK}

It is at this stage where a person uses the Boot Wizard to create the network boot disk. Due to the larger sizes of the files and because of the old hardware we have, the boot disk is composed of two floppy disks, one to store network drivers that load on boot and the second to store Ghost executable [9]. If one clicks on the start button, all programs and then chooses Symantec Ghost and from Symantec ghost chose Ghost Boot Wizard, the following screen will appear from the server.

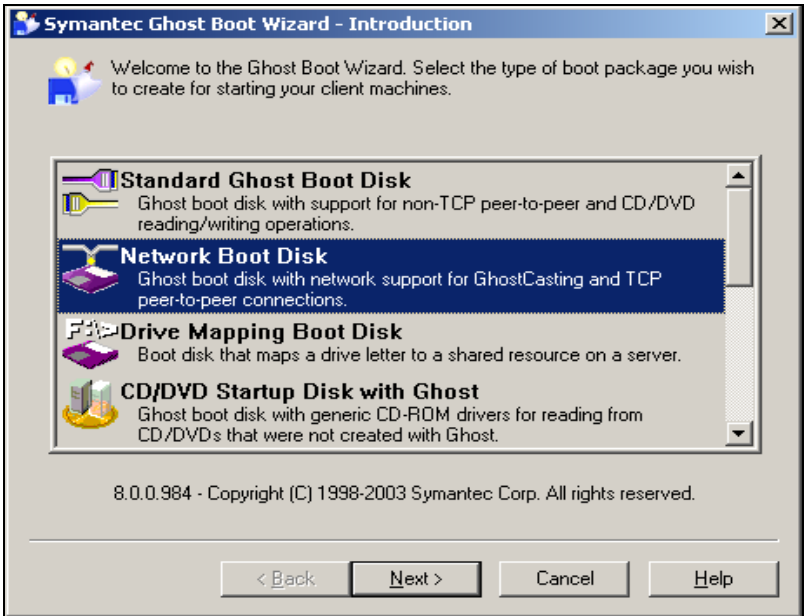

Figure 2: Symantec Ghost Boot Wizard

Figure 2 shows the Boot wizard where one can choose the type of disk he/she wants to create. For this project Network Boot Disk was chosen as is highlighted in the above figure. From this stage one should click on next and the following figure will show:

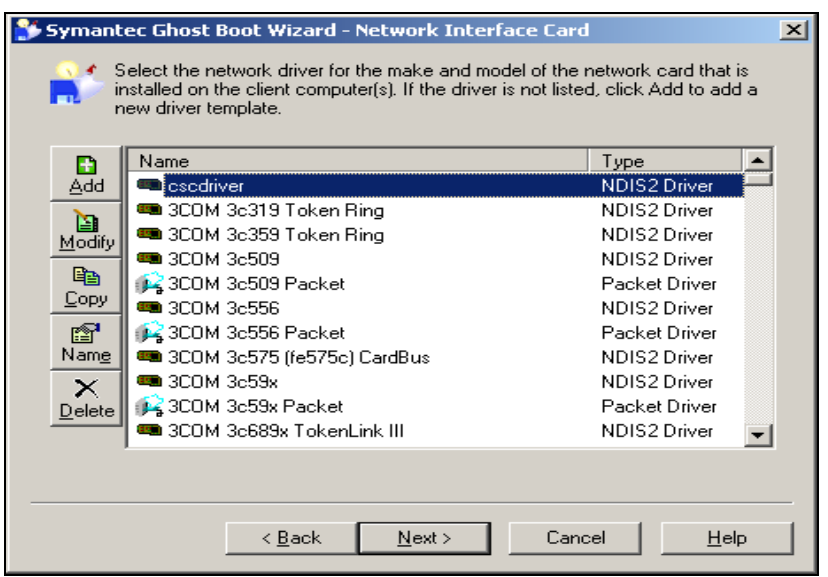

Figure 3: Network Interface Card

In the above figure one can chose the Network interface card of the clients he wants to Ghost. For this project as shown in the figure cscdriver was chosen [8]. Here one can choose any option as long as it is compatible with the clients that will be ghosted. Also, if it is not shown on the list it can be added by clicking on the left button labeled "Add". The one used here was not on the list and it was therefore added. After that click next to see the following screen: 


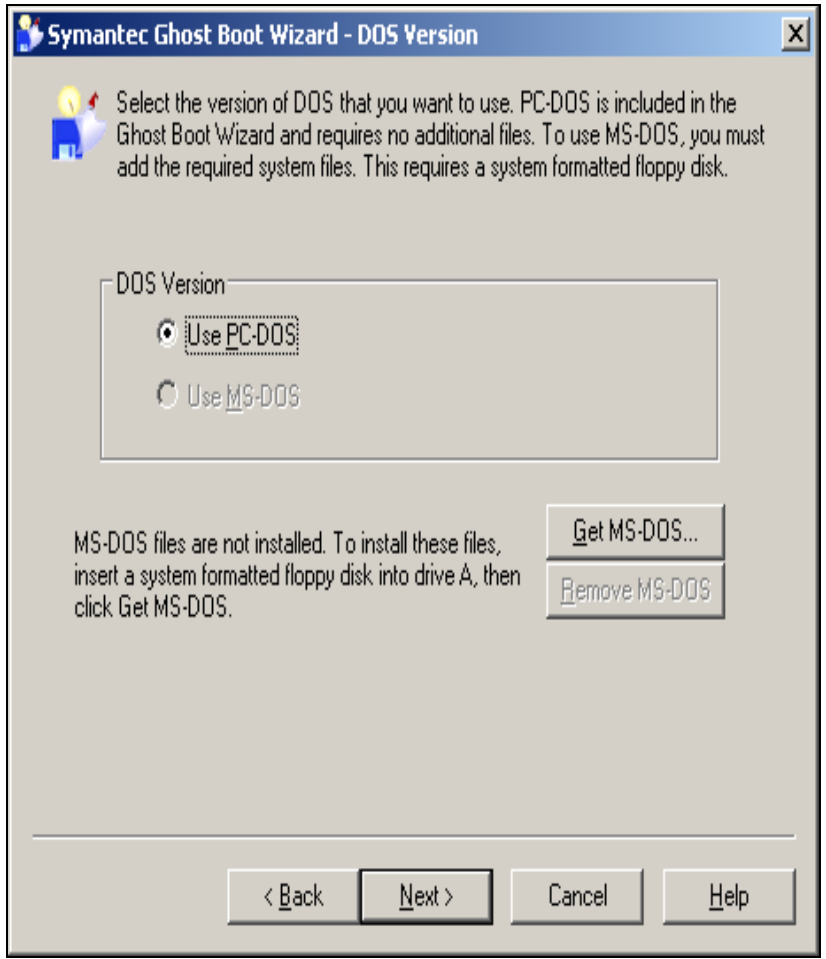

Figure 4: Dos Version

Most of the Symantec ghost software's use the Dos version and that is why it comes as a default choice on this stage. If one wants to use MS-DOS, he/she can do so by clicking the button labeled Get MS-DOS. These require the use of the formatted floppy disk and then click the check box labeled Use MS-DOS. It is also possible to remove the MS-DOS option if it already installed and it is not needed [10]. By clicking next one will get the following screen:

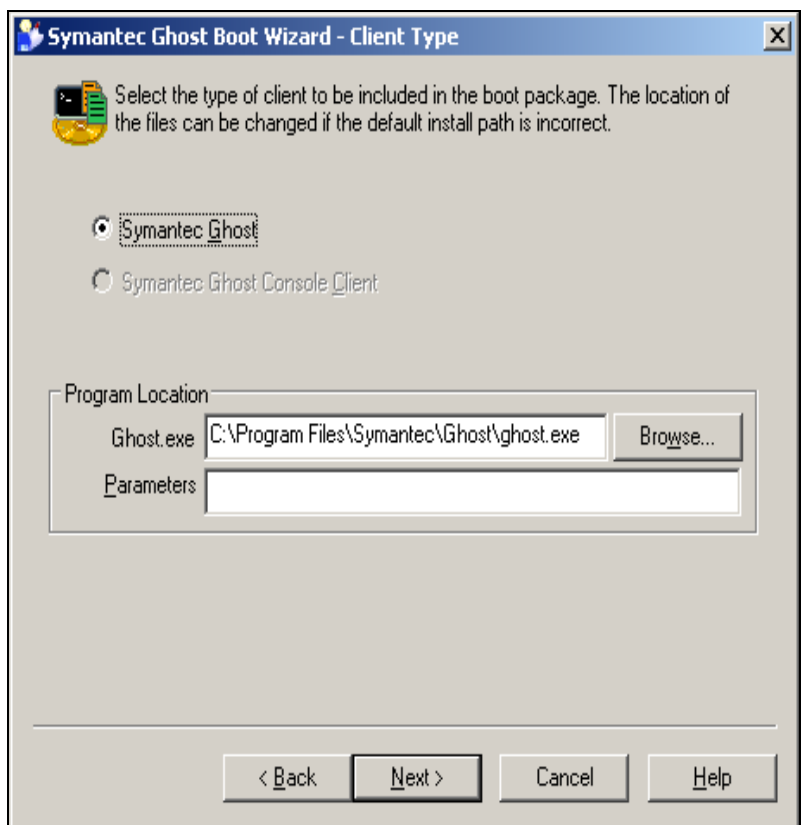

Figure 5: Client Type

From the above figure, this is where one can select the type of client to be included in the boot package and Symantec Ghost is chosen for this particular purpose. This figure also shows the location of the program and this location will later be used to store the image created. By clicking "next", the following screen will appear:

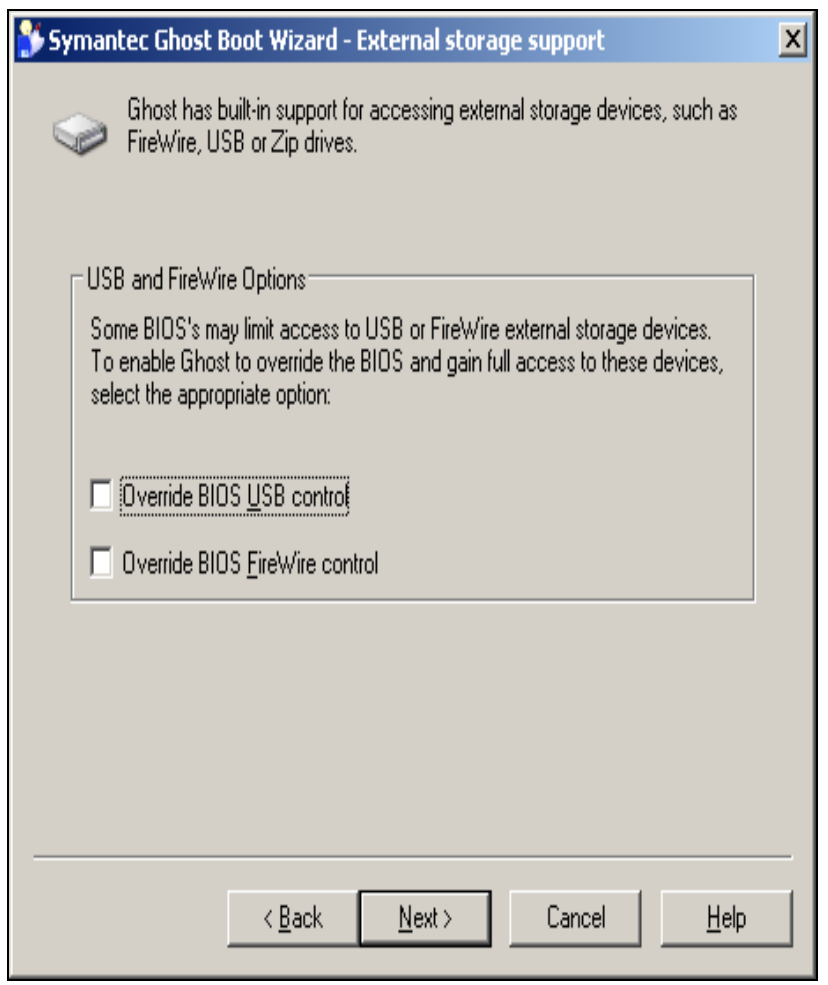

Figure 6: External Storage Support

The above figure allows the external storage medium such as USB's and CD's to be included in the image, especially if a person has some valuable files stored on a USB [14]. To enable Ghost to get full access to these devices click on the two check boxes to override BIOS. Click next to see the following screen:

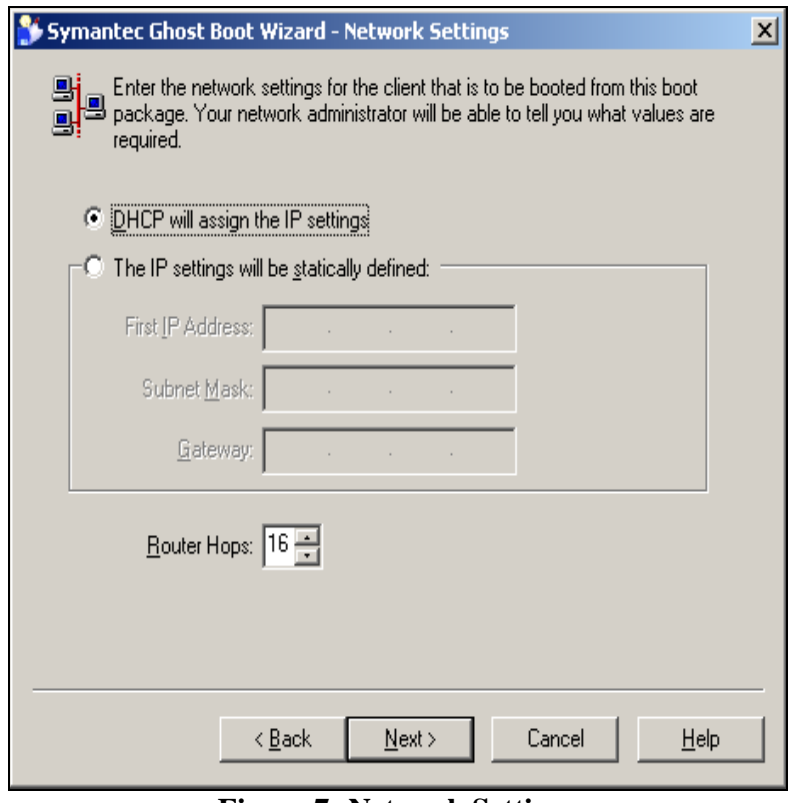

Figure 7: Network Settings

The above figure allows a person to choose from the dynamic or static allocation of IP addresses of the clients. Therefore dynamic allocation is chosen in this case as it is easy to work with it. A person does not have to remember all the IP 
addresses. The server searches for them automatically and adds them on the list [13]. The router hops is set to maximum as it may take too long for other clients to connect. Click next to see the following screen:

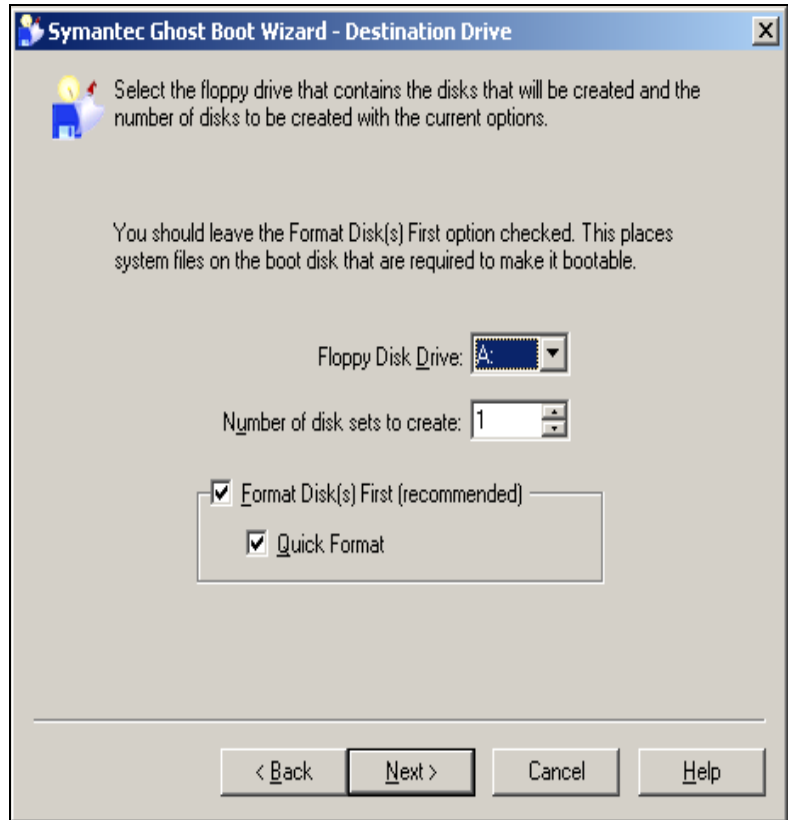

Figure 8: Destination Drive

Figure 8 above allows a person to select the correct destination of the bootable package and also to choose the number of floppy disks to be used. It also provides two check boxes that must be checked in order to format the disk. If these are not checked a person will not be able to create the boot disk. Even if one uses two floppy disks, when inserting the second disk Symantec Ghost will allow that person to format the second disk [15]. The quick format option helps to speed up the process as it is much faster than full format. Then click next to see the following screen:

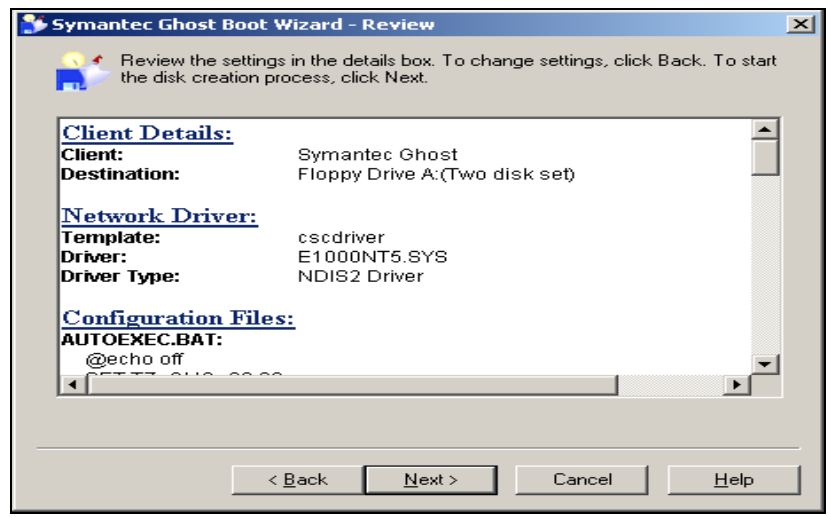

Figure 9: Review of Settings

The above figure shows a detailed review of all the settings that have been chosen. Starting by the client details, network driver details and then the configuration files details [16]. There is nothing much on this stage, it is only for a person to read and confirm that all the details are correct and that everything required for the Boot Disk is included. Click next to see the following screen:

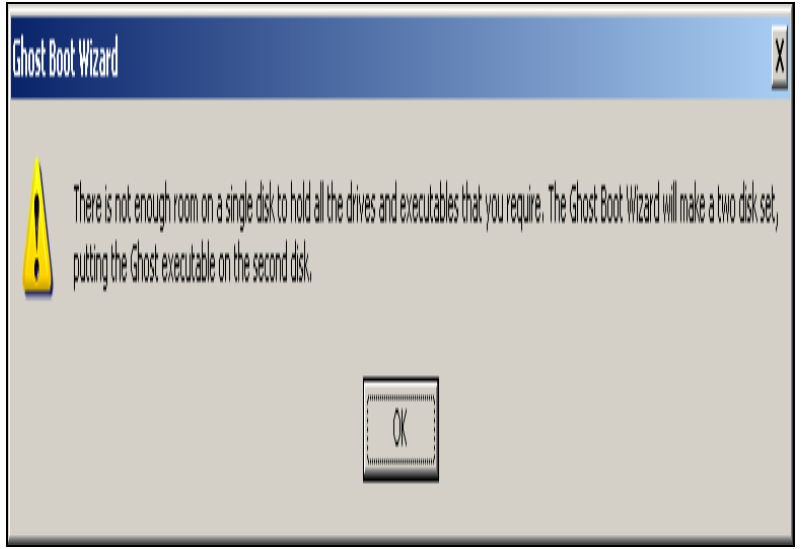

\section{Figure 10: Alert Message}

This is just a message alerting a person that two disks are needed. Click Ok for the following screen:

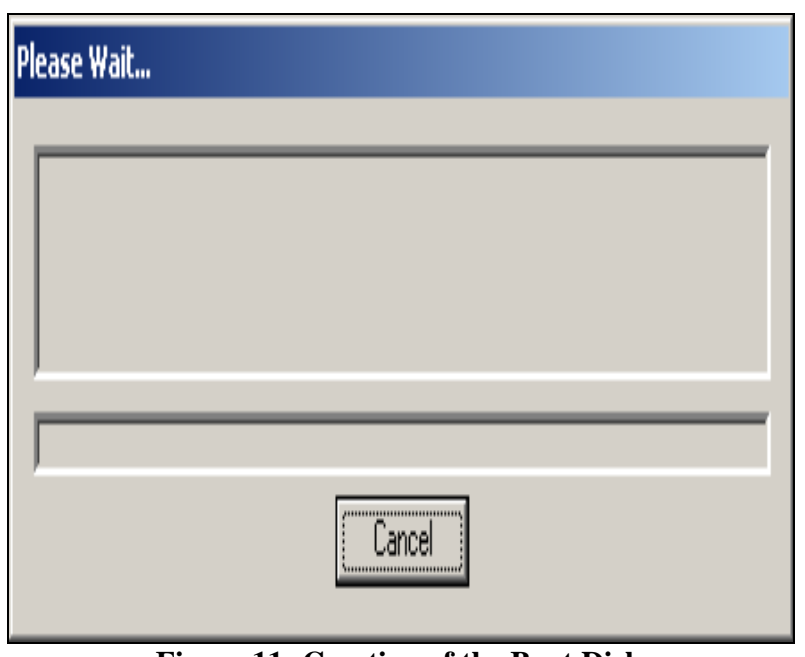

Figure 11: Creation of the Boot Disk

Figure 11 above shows the progress of the boot disk that is being created. But because the disk has not yet been formatted this will be disturbed by the following message:

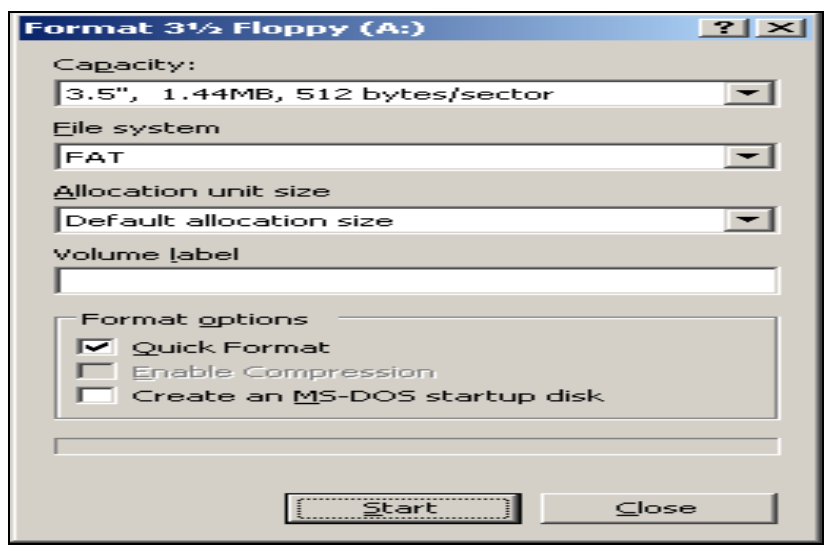

Figure 12: Format the Floppy Disk

This figure let a person to click on start to start formatting the floppy disk. Once this is complete it will return back to show figure 8. As is shown in the following figure, the creation of the boot disk is in progress after formatting the floppy. After the process in figure 13 below has finished, the screen in figure 14 will appear asking a person to click on finish to end the session. This stage will be the last before completion of 
the process. The image will be stored in the location specified. Then a person has to give a name to the session and it must be written down as it will be used for future connections between server and a client.

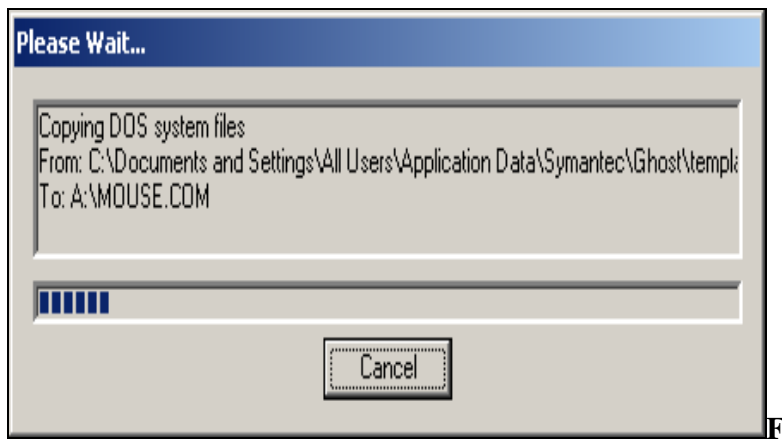

igure 13: Creation of the Boot Disk

By clicking enter after this stage; the screenshot given in figure 14 below will appear. This stage confirms the end of the process. Once a person clicks on "finish", it means the Ghost Boot disk has been created successfully.



igure 14: Process completed

\section{GHOST IMAGES CREATION}

It is at this stage where the actual Image will be created using Symantec GhostCast Server. The image will be stored in the location specified above in figure 8 . This location is copied then paste on the line that says Image File. Then a person has to remember to give a name to the session and it must be written down as it will be used for future connections between server and a client [17]. After opening GhostCast Server one should be able to see the following screen:

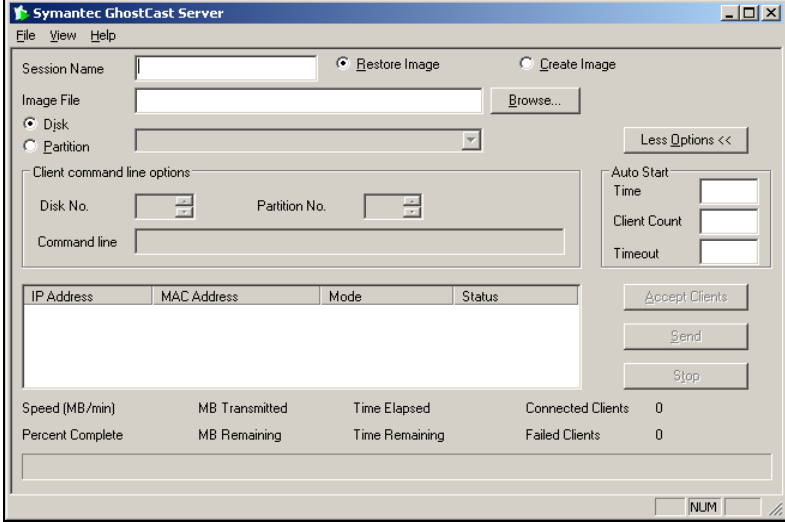

Figure 15: Symantec GhostCast Server

As shown in the above figure the Symantec GhostCast server has both Restore Image and Create Image options. Therefore for this stage of the project Create Image should be chosen. After the client has been installed all the applications and software needed by other computers where the image will be deployed, it is where a person click on the button labeled Accept Clients. This button can only be highlighted if there is a session name, Image file and both the server and the client are on the same subnet [18]. On the client side one has to choose Unicast and provides the same session name. Therefore the image creation will begin. This is shown clearly on the following figures.

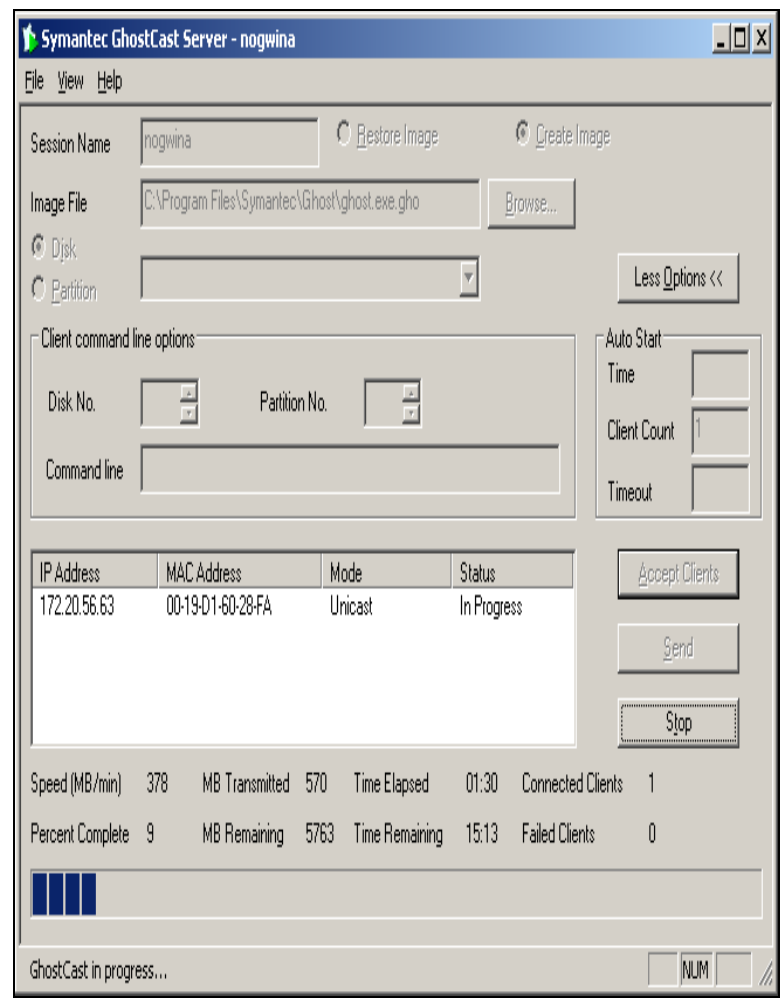

Figure 16: Image Creation in Progress

In figure 16 above, the image creation is in progress and it shows the server side. In this case, the session has been given a name nogwina and the client has established the connection with the server. Session name is nogwina and the IP address of the client is shown as well as the Media Access Control (MAC) address. It also shows the time elapsed and the time remaining for the image to finish. Before one sees this screen, few operations on the client have to be performed. If ok is 
clicked in figure 10, then the above screen in figure 16 will appear and this is where one can choose GhostCast and then unicast to start the session [19]. The client is joining the session named nogwina and the discovery method is left to be automatic because when the boot disk was created, dynamic allocation of the IP addresses was chosen. The above figure allows a person to confirm the drive or partition to be copied and it also shows the size of the driver. At this stage one has to choose not to compress the image by clicking No and then proceed to the next screen. If one need to compress the image fast or high can be chosen. One should click "Yes" at this stage to proceed with the image creation. The above figure shows the progress of the image creation from the client side and that the connection has been established between the server and the client. Figure 16 above shows that the image was completed successfully and is now ready to be deployed to other clients.

\section{GHOST SOFTWARE OPERATIONS}

This research project makes use of the Ghosting software, in particular Symantec Ghost. This software may sometimes be used as a backup program although it is not meant specifically for this. It can actually restore the entire disk. This software also allows its users to store the image for later reuse. It also supports multicasting, meaning one image can be deployed over a number of computers as long as they meet the hardware and software requirements such as having the same NIC. It also offers support for external media such as CD and USB whilst it also has the ability to read and write images to and from NTFS partitions. The previous section dealt with the creation of the image and at this stage it is where one should look at specific requirements for each computer that needs to be reimaged. The clients that are going to receive the image may be different and therefore it might not be easy to just deploy the image over the network. The most important thing one should look at before deploying the image is that the client computers are the same model or not and also the NIC is the same or not. If the NIC is not the same, a boot disk for each client that uses a different NIC has to be made separately. This will allow the different clients to be booted from its own boot disk that contains the NIC similar to the one of its own. Things become simpler if the client computers are all the same, only one boot disk is made for all of them. The following figure shows the GhostCast server establishing a connection with the client for restoring or deploying the image over the network.

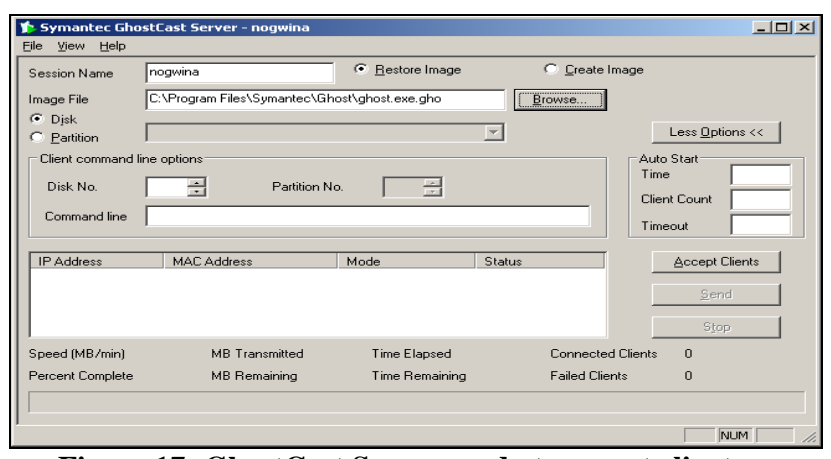

Figure 17: GhostCast Server ready to accept clients

In the previous sections, the option that was chosen in the GhostCast server was "create image" and here "restore image" is chosen because the image is now sent to the desired clients. The name of the session is also provided and it should be the same as the one that will be chosen for the client. After these few operations, one should click on "accept clients" and wait for all the clients to connect. After all clients have connected then click "send" to start deploying the image over the network. At this stage one should start seeing the time elapsed and the remaining time for the deployment to finish. This is only seen on the server where the image was stored after being created. The number of clients connected is also shown in the GhostCast server. All the operations described above only take place on the server. The computers used as clients should have a matching Network Interface Card (NIC) from the Ghost Boot Wizard [20]. Above all, for the deployment of Ghost images to be successful, the Symantec Ghost version has to be registered or licensed.

\section{CONCLUSION}

This paper has thoroughly outlined the technical concept of ghosting and gave an exhaustive overview of how this process can be implemented using Norton symantec ghost software for windows and open source platforms. Ghost imaging, using ghosting software has been defined as a method of converting the contents of a hard drive including its configuration settings and applications into an image, and then storing the image on a server or burning it onto a CD. When contents of the hard drive are needed again, ghosting software converts the image back to original form. Companies, institutions and organisations alike can use ghost imaging when they want to create identical configurations and install the same software on numerous machines. For instance, if a company needs to dole out 100 laptops to its employees, then instead of manually setting configurations, and installing applications on each machine, ghosting software (usually contained on a floppy or hard disc) will retrieve the ghost image from the server, convert it into its original form, and copy it onto the PC. By so doing, many clients systems can be installed with applications and services at once reducing the load of having to perform client by client installation or upgrade. This therefore ensures that this process and the ultimate goal of software deployment is thus achieved in a cost and time effective manner.

\section{REFERENCES}

[1] Bowman, M., Debray, S. K., and Peterson, L. L. 2012. Acronis True Image Home 2012 (2012). Top-tenreviews. Accessed online from: http://disk-imagingsoftware-review.html

[2] Agarwal A. RichCopy: The Only File Copying Utility You'll Ever Need.Accessed online from: http://www.labnol.org/software/richcopy-file-copying/

[3] Agarwal A. RichCopy: The Only File Copying Utility You'll Ever Need.Accessed online from: http://www.labnol.org/software/richcopy-file-copyingtool-for-windows/8035/

[4] Ashraf (2009). 8 free software to image/backup/ghost your computer (free alternatives to Acronis True Image, Norton Ghost, etc.). Accessed online from: http://dottech.org/6194/7-free-software-toimagebackupghost-your-computer-free-

[5] Alternatives-to-acronis-true-image-norton-ghost-etc/ Anebeg (2008). Successfully Deploy a Ghost Image to Many Computers at the same Time. Accessed online from: http://anebg.net/2008/07/01/to-many-computers 
[6] image-to-many-computers-at-the-lab http://anebg.net/2008/07/01/successfully-deploy-a-ghostimage-to-many-computers-at-the-lab

[7] Norton Ghost History. Accessed online from: http://www.softpanorama.org/Windows/Ghosting/ghost_ history.shtml

[8] Flanerin B.and Platedf, R. 2011. Windows Ghosting input. In Proceedings of the Conference on Human Factors in Computing Systems, Seychelles, 2011

[9] Tedfin, P. 2012 Ghosting and Cloning Design. AK Peters Ltd.

[10] Sannella, M. J. 1994 Constraint Satisfaction and Debugging for Interactive User Interfaces. Doctoral Thesis. UMI Order Number: UMI Order No. GAX9509398., University of Washington.

[11] Cnet Download (2010). Tera Copy.Accessed online from: http://download.cnet.com/TeraCopy/3000-2248_410671835.html

[12] DIY Computer Repairs (2007). Making a Symantec Ghost Image Using Symantec Ghost.Accessed online from: http://www.diy-computer-repair.com/symantecghost-image.html

[13] File Guru. (2010). PC Disk Clone X 11.0.Accessed online from: http://www.fileguru.com/PC-Disk-Clone$\mathrm{X} /$ info

[14] Future Systems (2012). Casper Complete PC Backup Protection.Accessed online from: http://www.fssdev.com/default.aspx?adc=1
[15] International Software Industry Association (2011). PC Disk Clone X 11.0.Accessed online from: http://www.softwaredownloads.org/windows/systemutilities/backup-restore/pc-disk-clone-x/

[16] Kierkels D. (2007). How to Create an Image Using Symantec Ghost, GhostCast Server.Accessed online from: http://ezinearticles.com/?How-To-Create-AnImage-Using-Symantec-Ghost,-Ghostcast-

[17] MFSN (2007). [WWW]. Multiboot CD's/DVD's Accessed online from: http://www.msfn.org/board/topic/107428-ghost-11-

[18] Microsoft (2012). How To Add a Boot Image to WDS Server (en-US).Accessed online from: http://social.technet.microsoft.com/wiki/contents/articles/ 11643.how-to-add-a-boot-image-

[19] Norton Ghost User's Guide (2003). Guide to Symantec Ghost 8.0.Accessed online from: $\mathrm{ftp} / / / \mathrm{ftp}$. symantec.com/public/english_us_canada/product s/ghost/manuals/symghost_8

[20] Navarana (2009). Create a Symantec Ghost Image for Hard Drive Cloning. Accessed online from: http://ulderi.com/my-itc-blog/2009/09/create-asymantec-ghost-image-for-hard-drive-

[21] Nerdyloft (2011). WDS: Boot Image Changes Not Happening for You?

[22] Ozzu Webmaster Forum (2003). Creating a Ghost Image for Windows XP or the Like. Accessed online from: http://www.ozzu.com/mswindows-forum/creating-ghostimage-for-windows-the-like- 